\title{
Genetic characterization of an aggressive optic nerve pilocytic glioma
}

\author{
Christopher S. Hong ${ }^{1} \cdot$ Greg Fliney $^{2} \cdot$ Adeniyi Fisayo $^{2} \cdot$ Yi An $^{3} \cdot$ Pallavi P. Gopal $^{4} \cdot$ Antonio Omuro $^{5}$. \\ Renelle Pointdujour-Lim ${ }^{2} \cdot$ E. Zeynep Erson-Omay ${ }^{1} \cdot$ S. Bulent Omay ${ }^{1}$
}

Received: 28 August 2020 / Accepted: 6 October 2020 / Published online: 24 October 2020

(c) The Japan Society of Brain Tumor Pathology 2020

\begin{abstract}
Optic nerve glioma (ONG) is a rare, typically slow-growing WHO I grade tumor that affects the visual pathways. ONG is most commonly seen in the pediatric population, in association with neurofibromatosis type 1 syndrome. However, sporadic adult cases may also occur and may clinically behave more aggressively, despite benign histopathology. Genetic characterization of these tumors, particularly in the adult population, is lacking. A 39-year-old female presented with 1 month of progressive left-sided visual loss secondary to a enhancing mass along the left optic nerve sheath. Initial empiric management with focal radiotherapy failed to prevent tumor progression, prompting open biopsy which revealed a WHO I pilocytic astrocytoma of the optic nerve. Whole-exome sequencing of the biopsy specimen revealed somatic mutations in NF1,FGFR1 and PTPN11 that may provide actionable targets for molecularly guided therapies. Genetic characterization of ONG is lacking but is needed to guide the management of these rare but complex tumors. The genomic alterations reported in this case contributes to understanding the pathophysiology of adult sporadic ONG and may help guide future clinical prognostication and development of targeted therapies.
\end{abstract}

Keywords Optic glioma $\cdot$ Genomics $\cdot$ Sequencing $\cdot$ Neurofibromatosis $\cdot$ Case report

\section{Introduction}

Optic nerve glioma (ONG) is typically a slow-growing glial tumor affecting the visual pathway. The pediatric population is most commonly affected in association with neurofibromatosis type 1 syndrome, but sporadic cases may also occur in both children and adults. Typically, ONG is characterized as World Health Organization (WHO) grade 1,

E. Zeynep Erson-Omay zeynep.erson@yale.edu

$\square$ S. Bulent Omay sacit.omay@yale.edu

1 Department of Neurosurgery, Yale School of Medicine, 300 Cedar Street, TAC S327, New Haven, CT 06511, USA

2 Department of Ophthalmology and Visual Science, Yale School of Medicine, New Haven, CT, USA

3 Department of Therapeutic Radiology, Yale School of Medicine, New Haven, CT, USA

4 Department of Pathology, Yale School of Medicine, New Haven, CT, USA

5 Division of Neuro-Oncology, Yale School of Medicine, Yale Cancer Center, New Haven, CT, USA but tumor grade may not necessarily predict clinical behavior [1-3]. Treatment is usually reserved for cases in which visual acuity is worsening or significant progressive tumor growth is observed on serial imaging [4]. The molecular characterization of these tumors, outside their typical association with neurofibromatosis type 1 syndrome, has been poorly described but could guide treatment decision-making. In this report, we describe an adult case of a sporadic ONG with progressive growth despite WHO I grade. Comprehensive genomic sequencing of the tumor and matching blood was performed, providing novel genetic insight into these rare but complex tumors and potential actionable genomic targets for molecularly targeted therapies.

\section{Case description}

A 39-year-old woman presented with 1 month of decreased visual acuity of the left eye (OS). Best-corrected visual acuity was 20/15 in the right eye (OD) and 20/80 OS. There was a left relative afferent pupillary defect. Fundoscopic examination revealed temporal pallor of the left optic disc. She reported an unspecified incidental abnormality on brain 
magnetic resonance imaging (MRI), 8 years prior. Repeat brain MRI revealed a heterogeneously enhancing mass along the left optic nerve sheath, suggesting optic nerve sheath meningioma versus ONG (Fig. 1a). Family history was notable for mother, diagnosed with glioblastoma.

The patient declined biopsy because of risk of optic nerve injury. She was empirically treated with focal radiotherapy, $50.4 \mathrm{~Gy}$ in 28 fractions to $95 \%$ isodose line. MRI obtained 4 months after radiation demonstrated increased tumor size (Fig. 1b) extending to the optic chiasm, coupled with leftsided headache and declining visual acuity to counting fingers vision OS. She described inferior nasal scotoma OD and Humphrey visual field 24-2 demonstrated mild nonspecific defects OD and generalized depression OS. A left optic nerve biopsy was performed through an eyelid-crease incision. Histopathology demonstrated WHO grade I pilocytic astrocytoma of the optic nerve. She underwent six cycles of adjuvant temozolomide (150-200 $\mathrm{mg} / \mathrm{m}^{2} \times 5 / 28$ days), which stabilized tumor growth for over 7 months after biopsy (Fig. 1c). Notably, chemotherapy was complicated by SARS-CoV-2 (COVID-19) infection requiring hospitalization, followed by full recovery.

\section{Genomic analysis}

Whole-exome sequencing (WES) was performed on the biopsy in accordance with an institutional review boardapproved protocol (Table 1). Somatic mutations were identified in NF1 (NM_000267:c.T4839G:p.Y1613X), a lossof-function mutation, FGFR1 (NM_023110:c.C1638G:p. $\mathrm{N} 546 \mathrm{~K}$ ), an activating mutation previously reported in glial tumors [5], and PTPN11 (NM_001330437:c.G214A:p. A72T), an activating pathogenic variant on the $\mathrm{SH} 2$ domain that may drive oncogenic signaling in cancers, including gliomas [6] No large-scale somatic copy number alterations were identified. BRAF/KIAA1549 fusion was absent by
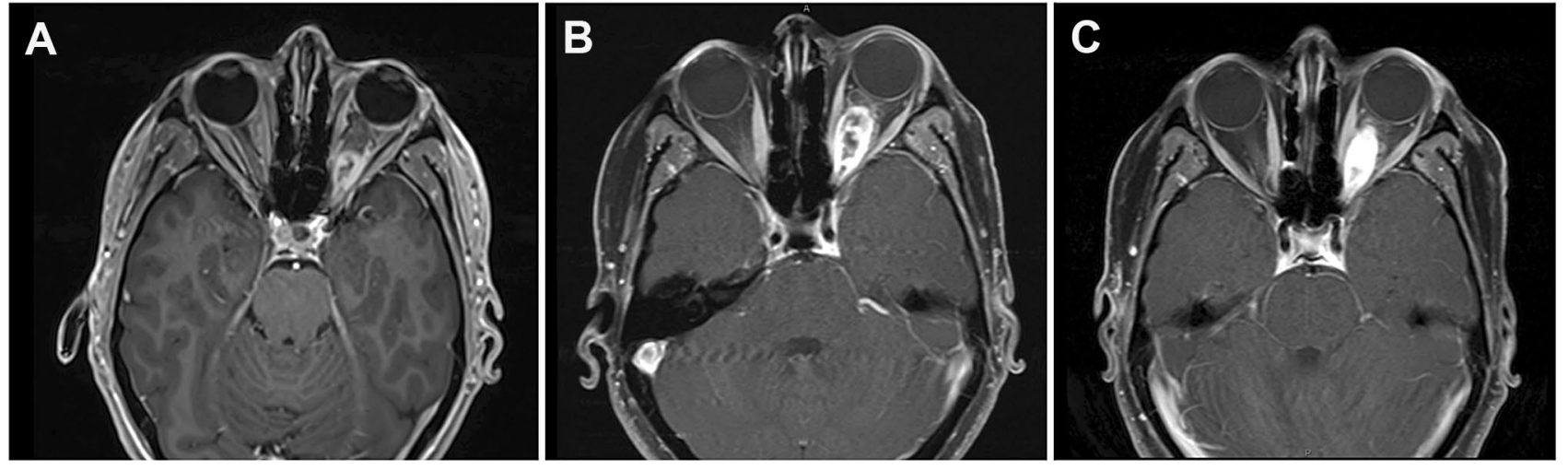

Fig. 1 Serial T1-weighted post-contrast brain MRI of the index patient. a Initial imaging demonstrates a left optic nerve lesion that (b) enlarged 4 months after completion of empiric radiation therapy. c
Surveillance imaging obtained after initiation of chemotherapy shows stabilization of the tumor, obtained 7 months after biopsy

Table 1 Summary of key genetic alterations derived from whole-exome sequencing of the biopsied tumor specimen

\begin{tabular}{|c|c|c|c|c|c|c|}
\hline Gene & Chromosome & Accesssion number & HGVS DNA reference & $\begin{array}{l}\text { HGVS } \\
\text { protein } \\
\text { reference }\end{array}$ & Variant type & Cited references \\
\hline NF1 & 17 & NM_000267 & c.T4839G & p.Y1613X & Nonsense & $\begin{array}{l}\text { Loss-of-function mutation driving ONG } \\
\text { formation in germline affected individu- } \\
\text { als and defines mesenchymal subtype of } \\
\text { sporadic glioblastoma }^{10}\end{array}$ \\
\hline FGFR1 & 8 & NM_023110 & c.C1638G & p.N546K & Missense & $\begin{array}{l}\text { Activating mutation prominent in low-grade } \\
\text { neuroepithelial and glioneuronal tumors } \\
\text { Activating mutation common in pilocytic } \\
\text { astrocytoma, including pilocytic ONG, and } \\
\text { may predict progression of WHO grade }{ }^{14}\end{array}$ \\
\hline PTPN11 & 12 & NM_001330437 & c.G214A & p.A72T & Missense & $\begin{array}{l}\text { Single case of pilomyxoid ONG reported in } \\
\text { patient with Noonan Syndrome }{ }^{16} \\
\text { Overexpression of PTPN11 enhances } \\
\text { tumorigenesis in glioma via activation of } \\
\text { PDGFR signaling }\end{array}$ \\
\hline
\end{tabular}


FISH. Additional WES of germline DNA was only notable for a somatic mutation in RYR1 (NM_000540:c.G7300A:p. G2434R), a variant as a well-established susceptibility allele for malignant hyperthermia (Fig. 2).

\section{Methods}

\section{Whole exome sequencing and analysis}

Genomic DNA from the tumor and blood were isolated followed by exome capture with IDT $\times$ Gen Exome Research Panel v1 with the additional spike-ins totaling $\sim 620 \mathrm{~kb}$ of RefGene coding regions. The sequencing was performed using Illumina NovaSeq6000 with $2 \times 100$ bp reads at Yale Center for Genome Analysis (YCGA). Downstream analysis starting from raw reads including alignment, duplicate marking, realignment, base quality recalibration was performed by following "GATK Best Practice" recommendations. Somatic single nucleotide variant (SNV), insertion/ deletions (INDEL) and copy number variations (CNV) were calculated as reported before [7]. Mean coverage of $300 \times$ and $133 \times$ was achieved for tumor and blood, respectively. Somatic WES analysis identified eight SNVs, only three of which were previously reported to be cancer related. No large-scale CNV events were identified.

\section{Discussion}

ONG most commonly presents as a WHO I pilocytic astrocytoma, diagnosed during childhood and often following a relatively benign course [8]. While rare, malignant ONG (WHO grade III-IV) is more commonly seen in adults and behaves much more aggressively with rapid visual decline followed by death within several months. Notably, a subset of ONG, typically diagnosed in adulthood, can behave aggressively, despite WHO grade I, as evidenced by continuous tumor growth despite radiotherapy in our case patient [1-3]. Comprehensive genetic analyses of these complicated tumors are needed to aid with prognosis and treatment, particularly for sporadic cases of ONG which may exhibit an unpredictable clinical course. However, few studies exist, possibly reflecting difficulty in obtaining tissue in this anatomical location [9].

WES of the index patient revealed a somatic NF1 mutation, well-recognized to propagate $\mathrm{ONG}$ in patients with germline NF1 mutations, but with an unclear role in sporadic ONG. Notably, this mutation was heterozygous, as no germline mutations or copy number alterations were detected to suggest loss of the other allele. However, somatic NF1 lossof-function mutations are frequently reported in gliomas, defining certain genomic subtypes [10] and heterozygous mutations have been described as sufficient for tumorigeneis in pre-clinical models $[11,12]$ and are also prevalent across many human cancers [13]. In a study of targeted sequencing for FGFR 1 activating mutations in 108 pilocytic astrocytomas, FGFR1 mutations were present in 13.9\% (15/108) of cases but notably showed a preoponderence within sporadic

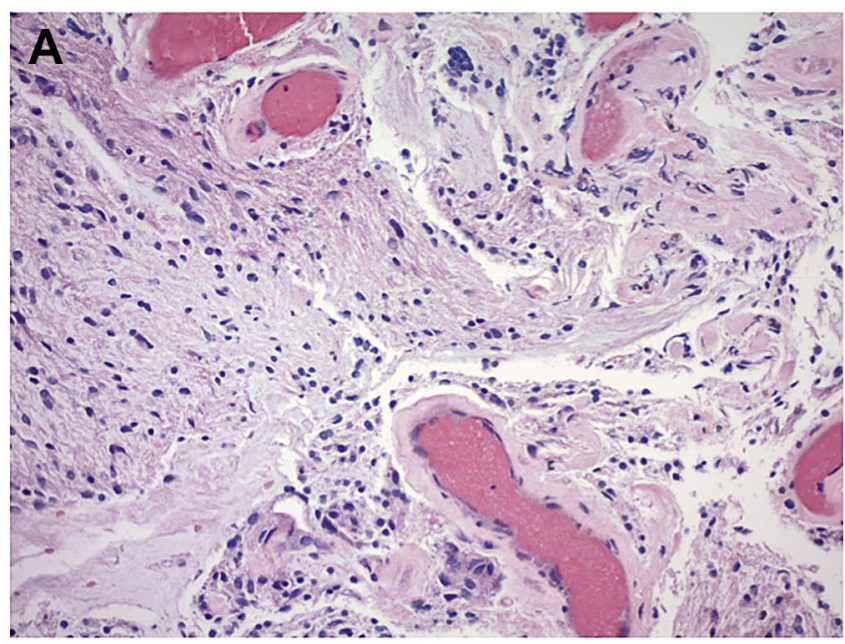

Fig. 2 Histopathology from the biopsied specimen. a Hematoxylin and eosin (H\&E) stained sections show a low grade glial neoplasm with features of pilocytic astrocytoma. The tumor has a vaguely biphasic pattern that includes denser regions and looser, myxoid and

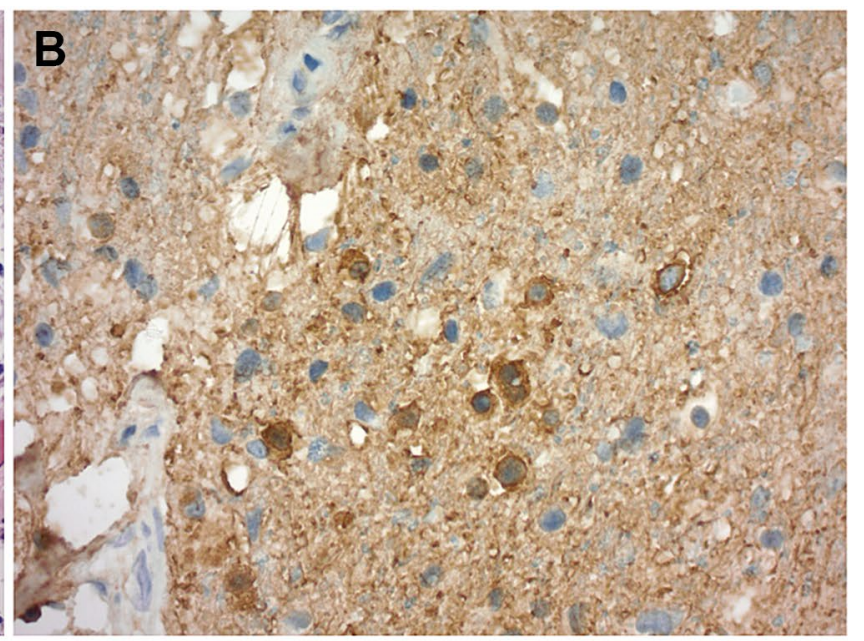

microcystic areas. b The neoplastic cells are GFAP positive by immunohistochemistry (IHC), and the Ki-67 proliferation index is $1 \%$ (not shown). H\&E image was taken at $200 \times$ magnification; GFAP IHC image was taken at $400 \times$ 
pilocytic astrocytomas for the optic pathway versus other locations (6/9 vs. 9/108, respectively) [14]. As such, our findings agree with the notion that FGFR1 mutations may be particularly prevalent in ONG and may have important implications for use of targeted anti-FGFR1 therapies in these tumors that are otherwise difficult to surgically access. Somatic mutations in PTPN11, also known as SHP-2, have not been reported in ONG, but mutations overlapping with the $\mathrm{SH} 2$ domain, as observed here, have been reported as key tumorigenesis drivers in other gliomas through activation of the AKT/mTOR signaling pathway $[6,15]$. Interestingly, a germline PTPN11-mutated ONG has been reported in a child with Noonan syndrome, characterized by germline gain-of-function mutations in PTPN11 and increased risk of myeloid and solid tumors [16]. Despite the family history of glioblastoma, no obvious pathogenic germline mutation was identified in our patient. Somatic PTPN11 mutations may also be more tumorigenic than germline mutations, due to greater gain-of-function [17], and is consistent with the more aggressive clinical course of our patient's tumor, despite WHO I grade. Importantly, given that PTPN11 mutations have also been reported in various oother cancers, including breast, liver, and gastric cancers, there are ongoing efforts to design selective pharmacologic inhibitors for targeted therapy $[18,19]$.

ONG remains rare but challenging to manage. Chemotherapy is often utilized for progressive ONG, usually defined as worsening visual acuity and/or significant increasing size on MRI. A variety of regimens have been reported, including temozolamide monotherapy, most of which has been studied in the pediatric population [20-22]. Surgical resection may be indicated in select patients but remains controversial, given the difficulty in accessing these lesions and proximity to nearby eloquent structures [23]. Likewise, the role of radiation remain unclear, particularly in the pediatric population where potential neurocognitive, cerebrovascular, and endocrine sequelae and risk of secondary malignancies exist [4]. Upfront radiation for progressive ONG in adults, as performed for our patient, has also been described in a handful of studies to varying degrees of success in local tumor control [24-26]. Notably, most of the studies on treatment for ONG has been in pediatric tumors, but the literature on management of sporadic adult ONG, which may arguably behave more unpredictably, is lacking. As such, increased molecular characterization is needed to predict cases warranting more aggressive first-line therapies, particularly in cases of sporadic ONG diagnosed in adulthood. This case provides unique comprehensive genomic study and describes key genetic alterations with potential actionable targets that may predict an aggressive clinical course despite standard treatment. Additional studies are required to investigate potential genomic targets for molecular-based therapies.
Acknowledgements None.

\section{Compliance with ethical standards}

Conflicts of interest None.

\section{References}

1. Shapey J, Danesh-Meyer HV, Kaye AH (2011) Diagnosis and management of optic nerve glioma. J Clin Neurosci 18(12):1585-1591

2. Pasol J, Sternau L, Luetmer P, Giannini C (2010) Rapid progressive unilateral visual loss in an elderly man. J Neuroophthalmol 30(2): 188-192

3. Bilgin G, Al-Obailan M, Bonelli L, Glasgow BJ, Vinters HV, Arnold AC (2014) Aggressive low-grade optic nerve glioma in adults. Neuroophthalmology 38(6):297-309

4. Farazdaghi MK, Katowitz WR, Avery RA (2019) Current treatment of optic nerve gliomas. Curr Opin Ophthalmol 30(5):356-363

5. Bale TA (2020) FGFR- gene family alterations in low-grade neuroepithelial tumors. Acta Neuropathol Commun 8(1):21

6. Liu KW, Feng H, Bachoo R et al (2011) SHP-2/PTPN11 mediates gliomagenesis driven by PDGFRA and INK4A/ARF aberrations in mice and humans. J Clin Invest 121(3):905-917

7. Kundishora AJ, Reeves BC, Nelson-Williams C et al (2020) Novel EWSR1-VGLL1 fusion in a pediatric neuroepithelial neoplasm. Clin Genet 97(5):791-792

8. Dutton JJ (1994) Gliomas of the anterior visual pathway. Surv Ophthalmol 38(5):427-452

9. Campbell AA, Gartrell-Corrado RD, Mansukhani M et al (2019) SETD2 mutation in an aggressive optic nerve glioma. JAMA Ophthalmol 2:6-10

10. Verhaak RG, Hoadley KA, Purdom E et al (2010) Integrated genomic analysis identifies clinically relevant subtypes of glioblastoma characterized by abnormalities in PDGFRA, IDH1, EGFR, and NF1. Cancer Cell 17(1):98-110

11. Gutmann DH, Loehr A, Zhang Y, Kim J, Henkemeyer M, Cashen A (1999) Haploinsufficiency for the neurofibromatosis 1 (NF1) tumor suppressor results in increased astrocyte proliferation. Oncogene 18(31):4450-4459

12. Ding H, Shannon P, Lau N et al (2003) Oligodendrogliomas result from the expression of an activated mutant epidermal growth factor receptor in a RAS transgenic mouse astrocytoma model. Cancer Res 63(5):1106-1113

13. Philpott C, Tovell H, Frayling IM, Cooper DN, Upadhyaya M (2017) The NF1 somatic mutational landscape in sporadic human cancers. Hum Genomics 11(1):13

14. Trisolini E, Wardighi DE, Giry M et al (2019) Actionable FGFR1 and BRAF mutations in adult circumscribed gliomas. J Neurooncol 145(2):241-245

15. Wang J, Cazzato E, Ladewig E et al (2016) Clonal evolution of glioblastoma under therapy. Nat Genet 48(7):768-776

16. Nair S, Fort JA, Yachnis AT, Williams CA (2015) Optic nerve pilomyxoid astrocytoma in a patient with Noonan syndrome. Pediatr Blood Cancer 62(6):1084-1086

17. Tartaglia M, Martinelli S, Stella L et al (2006) Diversity and functional consequences of germline and somatic PTPN11 mutations in human disease. Am J Hum Genet 78(2):279-290

18. Liu WS, Yang B, Wang RR et al (2020) Design, synthesis and biological evaluation of pyridine derivatives as selective SHP2 inhibitors. Bioorg Chem 100:103875 
19. Chen YN, LaMarche MJ, Chan HM et al (2016) Allosteric inhibition of SHP2 phosphatase inhibits cancers driven by receptor tyrosine kinases. Nature 535(7610):148-152

20. Packer RJ, Ater J, Allen J et al (1997) Carboplatin and vincristine chemotherapy for children with newly diagnosed progressive lowgrade gliomas. J Neurosurg 86(5):747-754

21. Massimino M, Spreafico F, Cefalo G et al (2002) High response rate to cisplatin/etoposide regimen in childhood low-grade glioma. J Clin Oncol 20(20):4209-4216

22. Gururangan S, Fisher MJ, Allen JC et al (2007) Temozolomide in children with progressive low-grade glioma. Neuro Oncol 9(2):161-168

23. Hidalgo ET, Kvint S, Orillac C et al (2019) Long-term clinical and visual outcomes after surgical resection of pediatric pilocytic/ pilomyxoid optic pathway gliomas. J Neurosurg Pediatr 13:1-8

24. Taveras JM, Mount LA, Wood EH (1956) The value of radiation therapy in the management of glioma of the optic nerves and chiasm. Radiology 66(4):518-528
25. Montgomery AB, Griffin T, Parker RG, Gerdes AJ (1977) Optic nerve glioma: the role of radiation therapy. Cancer 40(5):2079-2080

26. El-Shehaby AM, Reda WA, Abdel Karim KM, Emad Eldin RM, Nabeel AM (2016) Single-session Gamma Knife radiosurgery for optic pathway/hypothalamic gliomas. J Neurosurg 125(Suppl 1):50-57

Publisher's Note Springer Nature remains neutral with regard to jurisdictional claims in published maps and institutional affiliations. 